\title{
Heterogeneous Recurrence Monitoring of Dynamic Transients in Ultraprecision Machining Processes
}

\author{
Chen Kan ${ }^{1}$, Changqing Cheng ${ }^{2}$ and Hui Yang ${ }^{1 *}$
}

${ }^{1}$ Harold and Inge Marcus Department of Industrial and Manufacturing Engineering, Pennsylvania State University, University Park, PA 16802, United States

${ }^{2}$ Systems Science and Industrial Engineering, State University of New York (Binghamton), Binghamton, NY 13850, United States

${ }^{*}$ Corresponding author:

Hui Yang, e-mail: huy25@psu.edu; voice: (814) 865-7397; Fax: (814) 863-4745 


\begin{abstract}
In-situ monitoring and control of process variations are important for quality assurance in ultraprecision machining (UPM) processes. Recent advancements in sensing and communication technology have fueled increasing interests to develop sensor-based monitoring approaches for anomaly detection in the UPM process. However, conventional approaches are limited in their ability to address the complex dynamics hidden in the nonlinear and nonstationary processes. As a result, it is difficult for them to effectively capture the process variations of UPM. This paper presents a new heterogeneous recurrence monitoring approach to detect dynamic transients in UPM processes. First, a high-dimensional state space is reconstructed from in-situ sensing signals. A Dirichlet process (DP) driven clustering approach is then developed to automatically segment the state space into local recurrence regions. Furthermore, a fractal representation is designed to characterize state transitions among recurrence regions and extract novel measures to quantify heterogeneous recurrence patterns. Finally, we integrate a multivariate control chart with heterogeneous recurrence features for in-situ monitoring and predictive control of the UPM process. Experimental results showed that the proposed approach effectively detects transitions with a small magnitude, i.e., $\rho=28$ to $\rho=27$ in the Lorenz system, and identifies the shift from stable cutting $(\mathrm{Ra}=35 \mathrm{~nm})$ to unstable cutting $(\mathrm{Ra}=82 \mathrm{~nm})$ in UPM processes with an average run length of 1.0. This paper presents a novel data-driven DP clustering approach to characterize heterogeneous recurrence variations and link with the quality of surface finishes in UPM processes. This new DP recurrence approach circumvents the need to empirically define local recurrence regions and is shown to have strong potentials for manufacturing process monitoring and control that will increase the surface integrity and reduce rework rates.
\end{abstract}

\title{
Key Words:
}

Dirichlet process, heterogeneous recurrence, nonlinear dynamics, process monitoring, ultraprecision machining 


\section{Introduction}

Rapid advancement of microelectronics and precision manufacturing have fueled the increasing demand of mirror finish surfaces, which have been widely used in diverse industries such as defense and aerospace. Ultraprecision machining (UPM) is a commonly used manufacturing process to generate such optical disks. The UPM is also known as diamond turning and is equipped with a single crystal diamond tool and cyclically cuts nonferrous alloys or composites to generate nanometric surface finish (i.e., surface roughness $R_{a}<50 \mathrm{~nm}$ ) [1]. As opposed to conventional machining, the depth of cut in UPM is within the range of 2 to $50 \mu \mathrm{m}$, which is of the same order of magnitude as the tool edge [2]. As such, UPM process is exceedingly sensitive to environmental instabilities such as vibrations from nearby machines, temperature variations and inconsistency of material microstructures. A miniature process shift will immediately cause the variations of cutting forces and impact the quality of surface finish, e.g., resulting in large roughness or defects. This, in turn, will waste expensive tools and raw materials and lead to rework. Therefore, increasing yields and reducing costs for UPM hinge on the in-situ process monitoring and realtime detection of process shifts.

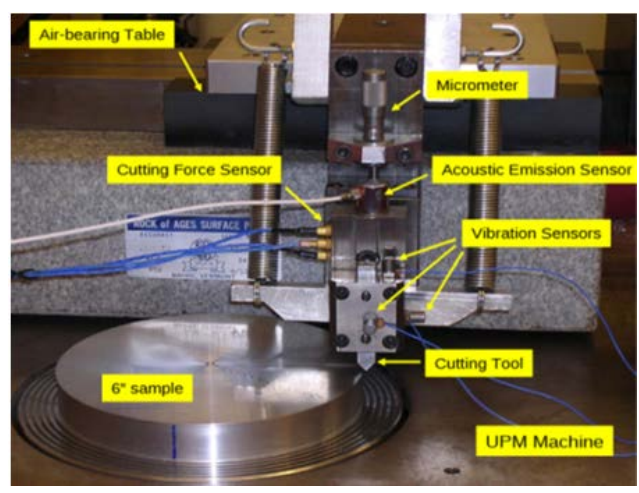

Fig. 1 UPM experimental setup equipped with cutting force, vibration and acoustic emission sensors.

In the literature, there are increasing interests at the development of data-driven modeling approaches for UPM process control. For example, Takasu et al. [3] focused on the deterioration of surface roughness generated from small vibrations. They developed a model to delineate the relationship between the surface roughness of workpieces and the amplitudes and phases of vibration. Cheung and Lee [4] simulated the three-dimensional topography of machined surface using a limited number of predicted roughness parameters, which helped determine the optimal cutting conditions and setup parameters. However, traditional modeling approaches are ineffectual in characterizing complex UPM processes, due to sudden surface finish variations and transient dynamics [1].

This present investigation focuses on advanced sensing technology for real-time monitoring and control of UPM process. As shown in Fig. 1, multiple sensors, including cutting force, vibration and acoustic emission (AE) are installed to extract useful information pertinent to process dynamics in UPM. Notably, in-situ assessment of UPM surface integrity has received increasing attention in recent years and is recognized as an emerging research area. For example, Dornfeld et al. [5, 6] pointed out the 
importance of tracking variations in process parameters such as material removal rate and tool conditions using high-frequency sensing. They specifically discussed the application of $A E$ sensors for the monitoring of UPM processes. Bukkapatnum et al. [7] investigated the low-dimensional chaos inherent to the lathe-turning process. Signals from cutting force and $A E$ sensors showed the turning operation exhibits low-dimensional chaos by the Lyapunov-exponent test.

However, nonlinear dynamics and nonstationary behaviors pose significant challenges on the development of new data-driven approaches for sensor-based monitoring and control of UPM processes. As the UPM process is highly sensitive to the variations of environmental conditions, the distribution of sensor signals is often non-Gaussian, nonlinear and nonstationary [1, 2, 8]. This, in turn, leads to sudden and sharp variations in the UPM surface morphology. Conventional change-detection approaches are more concerned about linear and Gaussian distributions, and most of them assume the system is in the steady state, i.e., stationary. Furthermore, signals collected from UPM processes are oftentimes contaminated by ambient noises. In other words, the signal-to-noise ratio is low and the frequency spectrum are with broadband characteristics [1]. Hence, traditional signal-processing techniques (e.g., Fourier analysis) are not well suited for analyzing UPM signals. An illustrative example is shown in Fig. 2. It may be noted that the frequency spectrum of vibration signals collected from stable (Fig. 2a) and unstable (Fig. 2b) processes registered similar dominant frequency components ( $380 \mathrm{~Hz}$ and $60 \mathrm{~Hz}$ ). Also, it is difficult to identify which peak corresponds to the machining process and which one is related to the extraneous factors. Therefore, conventional monitoring approaches are not well suited for capturing complex dynamics hidden in the nonlinear and nonstationary UPM processes.

(a)

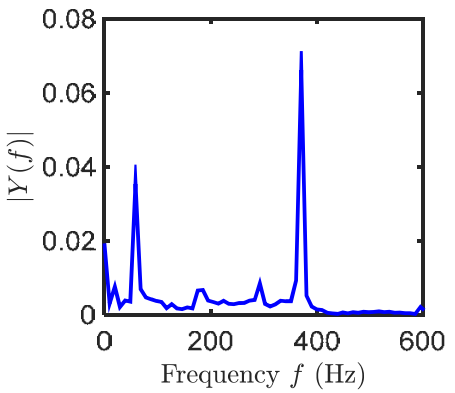

(b)

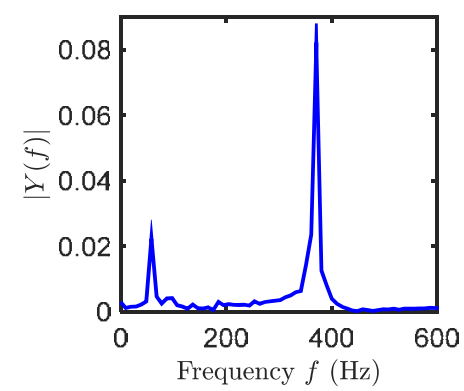

Fig. 2. Frequency spectrum of vibration signals in UPM, with major frequency components at $380 \mathrm{~Hz}$ and $60 \mathrm{~Hz}$ for (a) stable cutting and (b) unstable cutting.

To address aforementioned challenges, researchers have developed complex models to extract features pertinent to nonlinear and nonstationary dynamics in high-precision manufacturing processes. Instead of directly extract statistical patterns of raw signals, Rao et al. [1] utilized neural networks and Bayesian learning for the detection of incipient changes in UPM processes. They extracted the parameters (i.e., weights and biases) of neural network as surrogates to represent process states and further designed Bayesian particle filter to continuously update these parameters based on the observed sensor measurement. Beyca et al. [2] developed a data fusion strategy to integrate heterogeneous in-situ 
sensor signals for the detection of incipient anomalies in UPM processes. In phase I, Bayesian nonparametric Dirichlet Process (DP) model was proposed for offline clustering of sensor signals, which are further represented by Gaussian mixture models. In phase II, evidence theory was used for real-time decision-level fusion of newly arrived sensor data. Wang et al. [9] reported a change-point detection method to identify intermittency in UPM processes and chemical mechanical planarization (CMP) processes. The state space of process dynamics was represented by a stochastic mixture of Gaussian clusters. Intra-cluster state evolution was then represented by a nonlinear stationary process and intercluster transitions were captured by a Markov model. Recently, Rao et al. [10] proposed a graphical approach for the quantification of UPM surface variations. Instead of using 1-D signals, this method constructed a graph from microscopic surface images. Representative features (i.e., Fiedler number) were extracted from the graph for the detection of defects such as pits, ridges, and scratches.
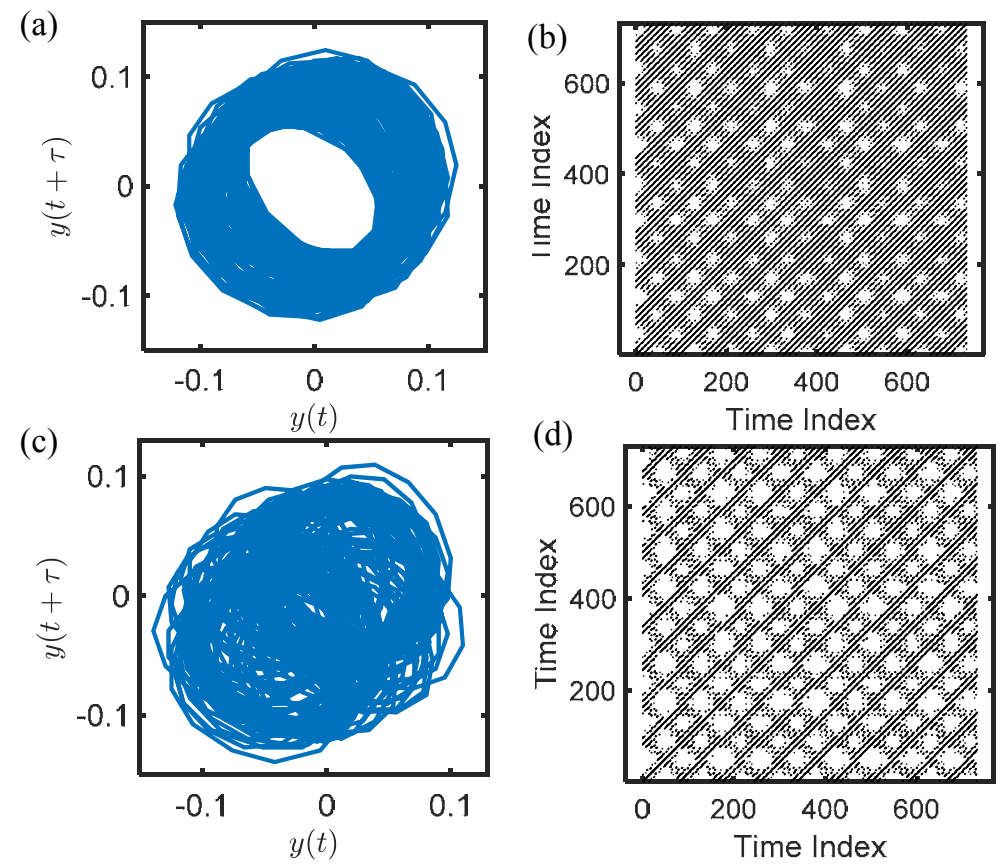

Fig. 3 The state spaces and recurrence plots for stable cutting ( $a$ and $b$ ) and unstable cutting (c and d).

It may be noted that nonlinear recurrence behaviors are common in real-world complex systems and have been widely used for the detection of process anomalies [11, 12]. Fig. 3 demonstrates 2D state space attractors extracted from vibration signals and corresponding recurrence plots for both stable (Fig. $3 a$ and $3 b$ ) and unstable (Fig. 3c and 3d) UPM processes. Notably, Fig. 3a shows a clear periodic circular trajectory but the attractor shown in Fig. $3 c$ is relatively chaotic. As a result, recurrence plots in Fig. $3 b$ and $3 d$ show distinct patterns. Recent investigations have shown that recurrence quantification analysis is an effective tool for characterising nonlinear and nonstationary dynamics [13, 14]. However, most of existing works focus on homogeneous recurrence analysis that characterize all recurring states in the same way using a Heaviside function. If the proximity of two states is greater than a predetermined threshold, it is color coded as a black dot in the recurrence plot. Otherwise, it is coded as white. As such, 
a black-and-white recurrence plot is obtained with black dots represent recurring states (see Fig. $3 \mathrm{~b}$ and 3d). Nevertheless, recurrence states may be heterogeneous (i.e., different types of recurrence behaviors) due to state properties, neighboring states and the evolving system dynamics [15, 16]. The intermittency and transient changes induced by underlying process shifts are more concerned with heterogeneous recurrence variations in the sensor signals. However, homogeneous recurrence analysis is limited in the ability to fully discern different kinds of recurrence behaviors in the UPM process.

To overcome the potential limitation of homogeneous recurrence analysis, our previous investigations developed heterogeneous recurrence methods for the characterization and modeling of nonlinear dynamic processes $[15,16]$. This new approach does not treat recurrence states in the same way. Instead, a segmentation scheme is developed to partition the state space into local regions. Different kinds of recurrence behaviors are then characterized in these local regions to reveal the underlying nonlinear and nonstationary dynamics. However, the number of sub-regions needs to be empirically defined in previous studies. In other words, the maximum capacity of Q-tree indexing is required to be defined beforehand. The effectiveness of heterogeneous recurrence analysis hinges on the optimal partition of states. Hence, extensive experiments were conducted to investigate how the number of subregions influence the performance of classification models [17]. The number of recurrence regions is varied, and the optimality is determined using the best classification accuracy in experiments. Nevertheless, such a trial-and-error strategy is not effective and requires expensive computation, which is not suited for in-situ process monitoring and control. Hence, there is a need to automatically identify the optimal number of local recurrence regions for the heterogeneous recurrence analysis.

This paper presents a DP-driven clustering approach to identify the optimal segmentation of local recurrence regions in the state space. As a nonparametric method, DP automatically optimizes the number of sub-regions from the probability distribution of recurrence states. The number of sub-regions need not be pre-defined by extensive experiments, which avoids expensive computation and makes heterogeneous recurrence monitoring more suitable for in-situ applications. This investigation provides a a novel sensor-based approach for in-situ monitoring and control of UPM processes. First, a highdimensional state space is reconstructed from in-process sensor signals. Then, a new DP-driven clustering approach is developed to recursively segment the state space into a hierarchical structure of sub-regions. Further, a fractal representation is designed to delineate transitions of recurrence states among local recurrence regions and extract useful information pertinent to heterogeneous recurrence patterns. To this end, a multivariate control chart is constructed to monitor heterogeneous recurrence quantifiers for real-time detection of defects in the UPM process. Experimental results on both simulated and real-world case studies show that the proposed approach effectively captures heterogeneous recurrence patterns in the transformed space and provides an efficient online monitoring scheme to detect process changes. 
Our contributions in this present investigation are as follows: 1) As opposed to empirically select the number of sub-regions, a nonparametric approach based on the Dirichlet process is introduced to identify the optimal number of sub-regions in a unsupervised way. This circumvents the expensive computation in previous studies and enables the implementation of heterogeneous recurrence analysis for online process monitoring; 2) We analyze heterogeneous recurrence behaviors in the vibration sensor signals of UPM and quantitatively characterize transient changes underlying the process dynamics. Furthermore, we theoretically formulate new control charts that detect the changes in the quality of surface finishes for the monitoring and control of the UPM process.

\section{Research Background}

Most of traditional statistical control charts are rooted in linear and Gaussian assumptions. However, sensor signals collected from UPM processes show nonlinear and nonstationary patterns. Traditional models are limited in their ability to capture the high-level nonlinearity, nonstationarity, and stochasticity that are inherent to UPM processes. One breakthrough in nonlinear theory is Poincaré's geometric analysis of dynamic systems in the state space. As shown in Fig. 3a and 3c, the state space is reconstructed from vibration signals based on Taken's embedding theorem [18]. Poincaré's recurrence theorem [19] further shows that if a dynamical system has a measure-preserving transformation, the trajectories of state space eventually reappear in the $\varepsilon$-neighborhood of former states with probability one. Recurrence is one of the most common phenomena in natural and engineering systems. In the literature, a graphical tool, i.e., recurrence plot, has been widely used to represent the nonlinear and nonstationary structures in the reconstructed state space. The recurrence plot characterizes the proximity of every state $\boldsymbol{x}(i)$ to all others $\boldsymbol{x}(j)$ by computing $R(i, j)=\Theta(\varepsilon-\|\boldsymbol{x}(i)-\boldsymbol{x}(j)\|)$, where $\Theta(\cdot)$ is the Heaviside function and $\|\cdot\|$ is a distance measurement (e.g., Euclidean norm). Fig. $3 b$ and $3 d$ show the recurrence plots for the state space trajectories in Fig. 3a and 3c, respectively. It may be noted that if two states are located closely to each other, the Heaviside function produces a value of one and the color code is black in the plot. Otherwise, the color code is white if they are located farther apart. The recurrence plot shows distinct topology and texture patterns. For example, the ridges locate transition states when the system switches between regimes in the state space. The parallel diagonal lines represent the near-periodicity of system behaviors.

In addition to graphical visualization, quantitative features are extracted to characterize the structures and nonlinear patterns in the recurrence plot such as chaos-order transitions and chaos-chaos transitions. Examples of recurrence quantifiers include determinism and laminarity. Determinism (DET) is the

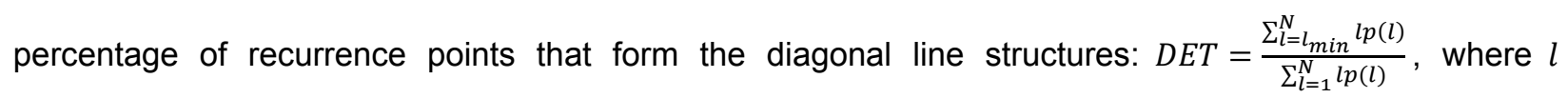
represents the length of lines that parallel to the diagonal line and $l_{\min }$ is the minimum length. $p(l)$ is the percentage of diagonal line segments with length $l$. Laminarity (LAM) calculates the percentage of 
recurrence points that form the vertical line structures: $L A M=\frac{\sum_{v=v_{\min }}^{N} v P(v)}{\sum_{v=1}^{N} v P(v)}$. Similarly, $p(v)$ is the frequency of vertical line segments with length $v$.

In the literature, recurrence quantification analysis was widely used for anomaly detection in manufacturing and healthcare. For example, Bukkapatnam and Cheng [11] developed a local Gaussian process model for time series forecasting by partitioning the nonstationary system into multiple nearstationary sub-systems. Rao et al. studied the recurrence behaviors of vibration signals for the characterization of underlying dynamics in the CMP process [20]. Recurrence quantifiers were shown to effectively characterize the CMP surface variations for the detection of process anomalies. In addition, Yang [13] and Chen [14] studied the relationship between recurrence patterns and cardiac disorders. Recurrence quantifiers were extracted from vectorcardiogram signals characterized in multiple frequency bands. These quantifiers effectively captured the transient and intermittent behaviors of cardiac electrical activity and lead to the accurate detection of disease-induced cardiac disorders. Recently, Yang and Chen [15] proposed to characterize heterogeneous recurrences patterns for monitoring and control of nonlinear dynamic processes. As opposed to homogeneous recurrences, heterogeneous recurrences were more concerned about the variations of state properties, neighboring states, and the evolving dynamics in the complex systems. Furthermore, Chen and Yang investigated heterogeneous recurrence behaviors in the continuous state space of a nonlinear dynamical system [16]. A multidimensional indexing method (namely, Q-tree indexing) was developed to iteratively decompose the state space until local regions reached the predefined maximal capacity. Such decomposition facilitated the clustering of states to delineate heterogeneous recurrence patterns for process monitoring and control.

In recent years, nonlinear dynamics have been increasingly studied to develop new statistical process control (SPC) approaches. With the rapid advancement of manufacturing technology, it is imperative for modern SPC to consider state-dependent sensor signals and nonlinear dynamics in the underlying process. For example, Singer and Ben-Gal [21] discussed the limitations of traditional SPC in handling nonlinear dynamics of sensor observations. In their approach, a Markov process was incorporated to improve the performance of feedback control in the classical funnel experiment. Also, Ruschin-Rimini et al. [22] developed a fractal-SPC approach that represented a discrete state space with iterative function mapping system and then extracted fractal dimensions as features for the monitoring of nonlinear and state-dependent processes. Recently, heterogeneous recurrence features were integrated with $T^{2}$ control chart for the detection of cardiac arrhythmic events [23]. The continuous state space reconstructed from Electrocardiogram (ECG) signals was partitioned using the Q-tree indexing. $T^{2}$ chart with heterogeneous recurrence quantifiers effectively detects the ectopic heart beats upon emergences. Furthermore, heterogeneous recurrence analysis was applied for the detection of obstructive sleep apnea [17]. The 3-D state space was reconstructed from RR intervals of single-lead ECG, which further decomposed into local sub-regions by the Q-tree indexing approach. Heterogeneous recurrence quantifiers were used as features to build the predictive model, which effectively classify sleep apneas from normal subjects. 
It may be noted that optimal segmentation of nonstationary state space into near-stationary ones is critical to delineate local recurrence patterns [24]. Our previous study [16, 17] introduced a multidimensional indexing approach to iteratively partition the state space into local recurrence regions. In addition to the expensive computation, empirical knowledge is required to predefine the number of subregions. Extensive experiments are often conducted to investigate how the number of sub-regions influence the performance of classification models. Such a trial-and-error strategy is not effective and is not suited for in-situ process monitoring and control. In the literature, there are different approaches used to identify optimal values to improve the results with machine learning techniques such as neural network [25] as well as computer simulation [26]. This present investigation is aimed at optimizing the clustering of local recurrence regions and further enhance the methodology of heterogeneous recurrence analysis for the monitoring of dynamic transients in the UPM processes.

\section{Research Methodology}

As shown in Fig. 4, a DP-driven self-organizing scheme is developed to partition the high-dimensional state space into local recurrence sub-regions. Then, a fractal representation is designed to delineate transitions of recurrence states in sub-regions and a new set of heterogeneous recurrence quantifiers are extracted. Finally, a Hotelling $T^{2}$ Chart is constructed with multivariate recurrence features for in-situ process monitoring and control. Notably, it is difficult to correlate time or frequency domain features that directly extracted from UPM signals with process anomalies. This is because UPM processes are highly nonlinear and nonstationary and traditional linear approaches are not well suited to extract features pertinent to the underlying transient dynamics in nonlinear and nonstationary processes.

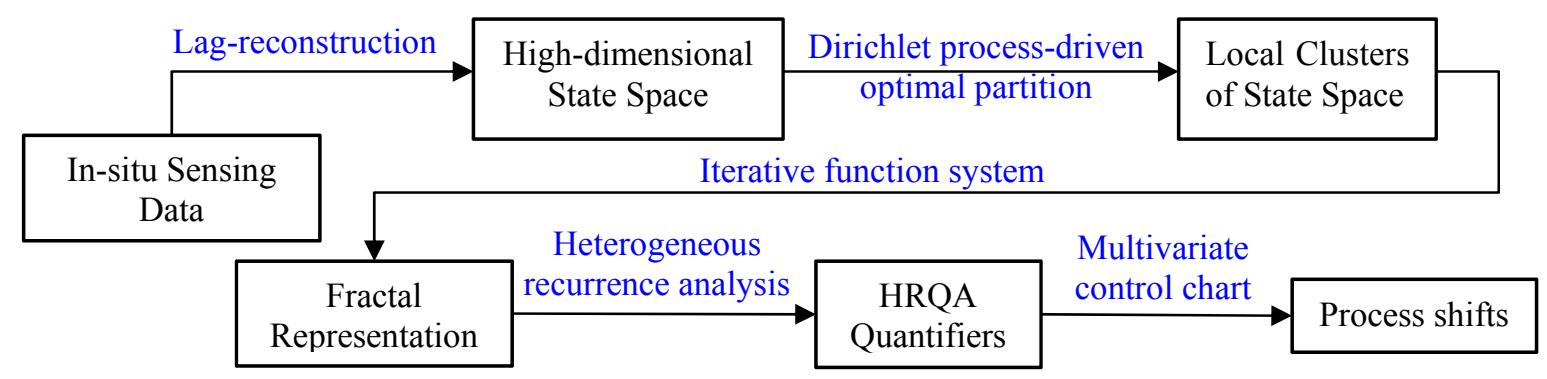

Fig. 4. Flow chart of the research methodology

\subsection{State Space Reconstruction}

The state evolution of continuous systems are usually described by a nonlinear stochastic differential equation $d \boldsymbol{x} / d t=F(\boldsymbol{x}, \boldsymbol{\theta}, \epsilon)$, where $F$ is a nonlinear function, $\theta$ is the parameter vector and $\epsilon$ is the noise. The solution $\boldsymbol{x}(t)=f(\boldsymbol{x}(0), t)$ delineates the state transition from an initial condition $\boldsymbol{x}(0)$, where $f(\cdot, \cdot)$ is the state transition function. Usually, the state $\boldsymbol{x}(t)$ is unobservable and the process is represented by the measured time series $\boldsymbol{y}(t)$, which is a function of $\boldsymbol{x}(t)$, i.e., $y=h(\boldsymbol{x})$. A time series from Lorenz model is shown in Fig. 5a. 
(a)

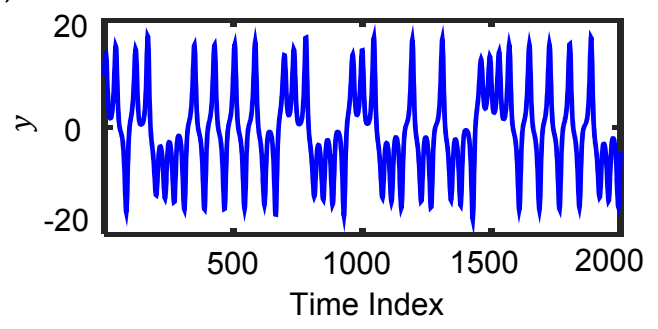

(b)

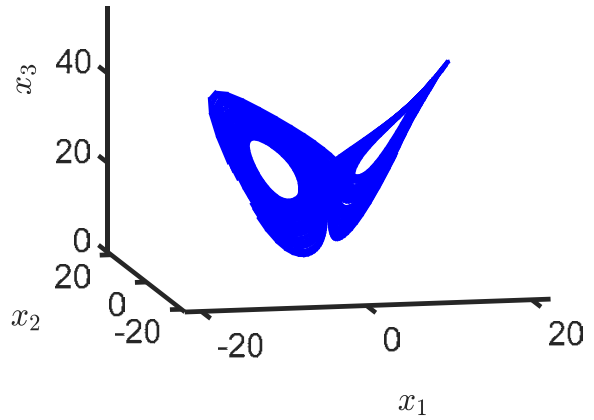

Fig. 5. (a) Original time series from Lorenz system; (b) Reconstructed 3-D state space vector (attractor) with $d=3$ and $\tau=10$.

When there is a small perturbation in $\boldsymbol{\theta}$, the process often undergoes abrupt changes and shows complex behaviors such as chaos and recurrences, resulting in the combined nonlinear and nonstationary dynamics in $\boldsymbol{y}(t)$. The dynamics manifest in the vicinity of an attractor, which is an invariant set defined in the $m$-dimensional state space. For time series $y_{1}, y_{2}, \ldots, y_{d}$, the state vector $\boldsymbol{x}(t)$ can be adequately reconstructed using a delay sequence of $\boldsymbol{y}(t)$ as [18]:

$$
\boldsymbol{x}\left(t_{i}\right)=\left[\boldsymbol{y}\left(t_{i}\right), \boldsymbol{y}\left(t_{i}+\tau\right), \boldsymbol{y}\left(t_{i}+2 \tau\right), \ldots, \boldsymbol{y}\left(t_{i}+(m-1) \tau\right)\right]
$$

where $m$ is the embedding dimension, which is optimally selected by the method of false nearest neighbor. The optimal time delay $\tau$ is selected to minimize mutual information function $M(\tau)$, which is defined as:

$$
M(\tau)=\int p(t, t+\tau) \log \frac{p(t, t+\tau)}{p(t) p(t+\tau)} d t
$$

where $p(t, t+\tau)$ is the joint density function, and $p(t)$ and $p(t+\tau)$ are marginal density functions of $\boldsymbol{y}(t)$ and $\boldsymbol{y}(t+\tau)$, respectively. With the lag-reconstruction, the original 1-D UPM signals are optimally projected into the $m$-dimensional state space for analyzing recurrence patterns.

\subsection{DP-driven Segmentation of Reconstructed State Space}

After reconstruction, it is necessary to partition the global state space into sub-regions to delineate local recurrence patterns. Such segmentation is equivalent to the clustering of states in the highdimensional state space. Simple clustering approaches, e.g., K-means clustering, are not suited for partitioning the state space. This is because K-means clustering requires all the previous states to compute the dissimilarity and update the objective function in each iteration. However, the state space is evolving over time. The computation will be highly expensive for K-means clustering and it is not suitable for in-situ process monitoring. Also, the results of K-Means clustering are not unique and they are varying with respect to time, which poses a great challenge for the following step of fractal representation. Some advanced approaches, e.g., multidimensional indexing used in [17], are also not well-suited for the clustering of states. In addition to expensive computation, most of those approaches require a pre-defined 
number of clusters, which usually is difficult to be determined beforehand for most real-world complex systems.

In this present investigation, the nonparametric Dirichlet process (DP) model is proposed to address these challenges. The basic idea of DP can be illustrated using the classic Chinese restaurant process. Generally, suppose that a Chinese restaurant has infinite tables and each table can be occupied by infinite customers. The customers are labeled by integer values. Customers sharing the same table follow a Gaussian distribution and those occupy different tables have distinct distributions. The $i^{\text {th }}$ customer opts to choose an already occupied table with a probability proportional to the number of customers at that table, or an unoccupied table based on the concentration parameter $\alpha$. By analogizing sensor observations as customers, sub-regions as tables in the Chinese restaurant process, the DP model partitions the global state space into sub-regions and assigns labels for new observations. The labels are obtained according to the assignment probability derived from the mean and covariance of each subregion, with each one following a multivariate Gaussian distribution. Assume that the state space is partitioned into $K$ sub-regions, the prior assignment probability according to Dirichlet distribution can be given as [27]:

$$
p(\boldsymbol{\pi})=p\left(\pi_{1}, \pi_{2}, \ldots, \pi_{K} \mid \alpha\right) \sim \operatorname{Dir}\left(\frac{\alpha}{K}, \ldots, \frac{\alpha}{K}\right)=\frac{\Gamma(\alpha)}{\Gamma\left(\frac{\alpha}{K}\right)^{K}} \prod_{k} \pi_{k}^{\frac{\alpha}{K^{-1}}}
$$

where $\alpha>0$ is a concentration parameter and $c_{i}$ is an indicator variable of data point $i$. As such, indicators of $n$ data points, i.e., $c_{1}$ to $c_{n}$, follow a multinomial distribution as $p\left(c_{1}, c_{2}, \ldots, c_{n} \mid \pi\right)=$ $\prod_{k=1}^{K} \pi_{k}^{n_{k}}$ (where $n_{k}$ is the number of data points in the $k^{\text {th }}$ sub-region and $\sum n_{k}=n$ ). This leads to the posterior distribution:

$$
p\left(\boldsymbol{\pi} \mid c_{1}, c_{2}, \ldots, c_{n}\right) \propto p\left(c_{1}, c_{2}, \ldots, c_{n} \mid \boldsymbol{\pi}\right) p(\pi)
$$

and it follows that:

$$
p\left(\pi \mid c_{1}, c_{2}, \ldots, c_{n}\right)=\frac{\Gamma(\alpha+n)}{\prod_{\mathrm{k}=1}^{\mathrm{K}} \Gamma\left(\frac{\alpha}{K}+n_{j}\right)} \prod_{k=1}^{K} \pi_{k}^{\frac{\alpha}{K}+n_{k}-1}
$$

It may be noted that the posterior also follows a Dirichlet distribution, i.e.,

$$
p\left(\boldsymbol{\pi} \mid c_{1}, c_{2}, \ldots, c_{n}\right) \sim \operatorname{Dir}\left(\frac{\alpha}{K}+n_{1}, \ldots, \frac{\alpha}{K}+n_{K}\right)
$$

Note that as the number of clusters $K \rightarrow \infty$, it is a Dirichlet process. The posterior indicator distribution can be updated using Gibbs sampling [27]:

$$
\begin{gathered}
p\left(c_{i}=k \mid \boldsymbol{c}_{-i}, \alpha\right)=\frac{n_{-i, k}}{n-1+\alpha} \\
p\left(c_{i} \neq c_{k} \forall k \neq i \mid \boldsymbol{c}_{-i}\right)=\frac{\alpha}{n-1+\alpha}
\end{gathered}
$$


where $n_{-i, k}$ is the number of data points in the $k^{\text {th }}$ sub-region before the assignment of data point $i$, and $\boldsymbol{c}_{-i}$ denotes the indicator vector for observations except for the point $i$. In other words, the new observations are most likely to be assigned to the sub-region with larger number of observations. Otherwise, they will be assigned to a new sub-region with a probability proportional to $\alpha$, i.e., the concentration parameter. A new state vector $x_{*}$ is assigned to the $k^{\text {th }}$ sub-region with probability given as $p\left(x_{*} \mid c=k, \boldsymbol{\mu}_{k}, \boldsymbol{\Sigma}_{k}\right) \sim \boldsymbol{N}\left(\boldsymbol{\mu}_{k}, \boldsymbol{\Sigma}_{k}\right)$. Here, the parameters $\boldsymbol{\mu}_{k}$ and $\boldsymbol{\Sigma}_{k}$ are the mean and covariance, with Gaussian distribution and inverse Wishart distribution prior, respectively. They can be updated after the assignment of new state vectors. Therefore, the assignment probability of $x_{*}$ to the $k^{\text {th }}$ sub-region can be derived as:

$$
p\left(c=k \mid x_{*}\right) \propto p\left(x_{*} \mid c=k\right) p(c=k)
$$

It may be noted that DP-driven partitioning of the state space reveals salient patterns relevant to the heterogeneous recurrences in the state space. Due to the nonparametric nature of DP, the shape and the number of clusters need not be known a priori. As shown in Fig. 6, the state space of the Lorenz system (see Fig. $5 b$ ) is partitioned into 7 sub-regions (represented by different colors), with each one following a

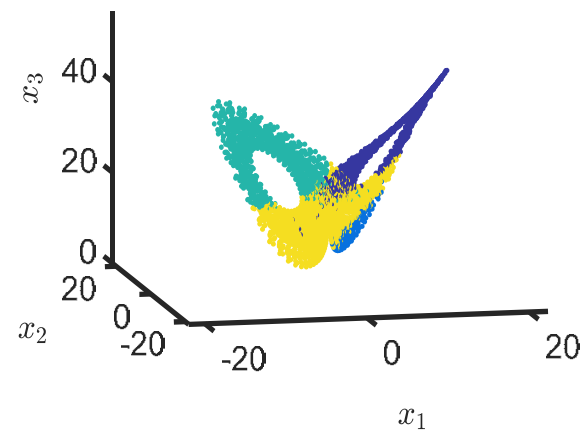

Fig. 6. Color-coded attractor (from Fig. 5b) showing the result of DP-driven state space segmentation. multivariate normal distribution.

\subsection{Heterogeneous Recurrence Analysis}

The DP generates an infinite number of sub-regions, whereas only up to $n$ are active ( $n$ is the total number of state space vectors). The remaining sub-regions will have zero observations. If the total number of active sub-regions is $N_{K}$, the time series of categorical variables can be represented as $c_{i}=k \in\left[1, N_{k}\right](i=1,2, \ldots, n)$ as states transit among the $N_{K}$ sub-regions. Here, an iterative function system (IFS) is introduced to characterize state transitions and represent heterogeneous recurrences in the time series of such categorical variables [16]. As such, it will facilitate the extraction of salient patterns of heterogeneous recurrences in the $2 \mathrm{D}$ fractal geometry. The basic idea of IFS is to sequentially map each state vector $\boldsymbol{x}(i), i=1, \ldots, n$ to an unique destination (i.e., a point $\left[C_{x}(i), C_{y}(i)\right]$ ) in the 2D coordinate system. Such mapping depends on all of previous states as well as the corresponding sub-region indicator $k$, as:

$$
\left[\begin{array}{l}
C_{x}(i) \\
C_{y}(i)
\end{array}\right]=\phi\left(k,\left[\begin{array}{l}
C_{x}(i-1) \\
C_{y}(i-1)
\end{array}\right]\right)=\left[\begin{array}{ll}
\alpha & 0 \\
0 & \alpha
\end{array}\right]\left[\begin{array}{l}
C_{x}(i-1) \\
C_{y}(i-1)
\end{array}\right]+\left[\begin{array}{l}
\cos \left(k \times 2 \pi / N_{K}\right) \\
\sin \left(k \times 2 \pi / N_{K}\right)
\end{array}\right]
$$

where $\left[\begin{array}{l}C_{x}(0) \\ C_{y}(0)\end{array}\right]=\left[\begin{array}{l}0 \\ 0\end{array}\right]$ and $\phi(k, \boldsymbol{d})$ is the mapping function. It may be noted that two states have the same address if and only if they share the same categorical variable, and all of their previous states are the same. However, this is not common in a nonlinear and nonstationary process. 

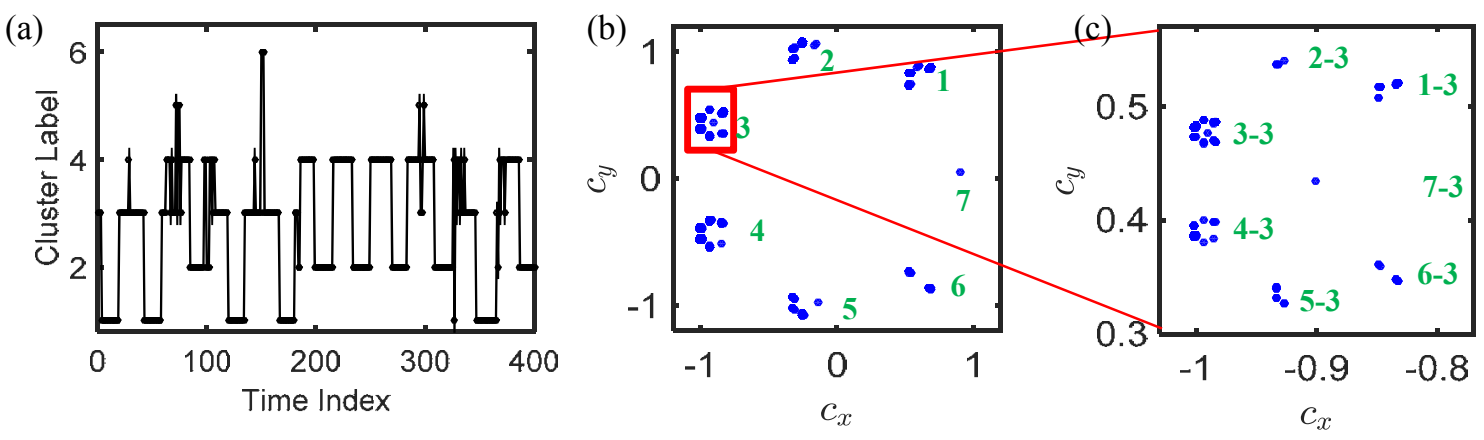

Fig. 7 (a) Time series of categorical variables showing state transitions, and the fractal representations of individual states (b) and two-state sequences (c) by zooming into sub-region \#3.

Fig. 7a displays the time series of categorical variables when states evolve among different subregions derived from DP. Fig. $7 \mathrm{~b}$ shows results of IFS for the time series in $7 \mathrm{a}$. There are 7 circles in Fig. $7 \mathrm{~b}$ and each of them represent one out of 7 categories. These categories are centered on 7 addresses that are uniformly distributed on the unit circle. As such, heterogeneous recurrences are effectively separated in the 2D graph in the level of 7 individual sub-regions. Notably, the density of each circle shows the number of observations in each sub-region. It may be noted that circles 3 and 4 are much denser than circles 6 and 7. Fig. 7c is obtained by zooming into circle 3 (marked by the red square in Fig. 7b). Every circle in Fig. 7c represents a transition from one sub-region (e.g., sub-region 1 to 7 ) to the subregion 3. Notably, circle 7-3 is missing in Fig. 7c. This show the transition probability is zero from subregion 7 to 3 in successive time indices. Similarly, the density of each circle in Fig. $7 \mathrm{c}$ demonstrates the frequency of a two-state sequence. By further zooming into any address in Fig. 7c, the three-state transition sequence can be obtained. In fact, the density and distribution of points in each circle characterize heterogeneous recurrence variations of the dynamics of state transitions among sub-regions. Therefore, the IFS transformation effectively reveals heterogeneous recurrence patterns in the time series.

However, the 2D fractal representation (Fig. $7 \mathrm{~b}$ and $7 \mathrm{c}$ ) cannot be directly used as quantitative features. Thus, quantifiers are further extracted to characterize heterogeneous recurrence patterns from the fractal representation. The clustered addresses are denoted as heterogeneous recurrence sets, $W_{k_{1}, k_{2}, \ldots, k_{L}} \equiv \phi\left(k_{1} \mid k_{2}, \ldots, k_{L}\right): x(n) \rightarrow k_{1}, \ldots, x(n-L+1) \rightarrow k_{L}$, where $k_{1}, k_{2}, \ldots, k_{L} \in\left[1, N_{K}\right]$ denotes the sequence of $L$-state transitions. Based on the set $W_{k_{1}, k_{2}, \ldots, k_{L}}$, three new quantifiers are defined: heterogeneous recurrence rate (HRR), heterogeneous mean (HMean), and heterogeneous entropy (HENT). HRR measures the percentage of recurrences in the fractal plot, which is analogous to recurrence rate in conventional Recurrence Quantification Analysis (RQA) measures. HMean provides general information about the average distance between elements in the set $W_{k_{1}, k_{2}, \ldots, k_{L}}$. HENT provides general information on the uncertainty in the heterogeneous recurrences. For more details, please refer to our recent work [19]. 


\subsection{Multivariate Control Chart}

Heterogeneous recurrence quantification describes multiple features pertinent to the dynamics of a complex system. The hypothesis test is to determine whether there is a significant mean shift in each feature vector $\boldsymbol{s}_{i}=\left[s_{i 1}, s_{i 2}, \ldots, s_{i p}\right]^{T}$ of the feature matrix $\boldsymbol{s}_{M \times p}=\left[\boldsymbol{s}_{1}, \boldsymbol{s}_{2}, \ldots, \boldsymbol{s}_{M}\right]^{T}$, where $p$ is the dimensionality of the heterogeneous recurrence features, and $M$ is the number of samples. Here, the feature vector $s_{i}$ is composed of the heterogeneous recurrence quantifiers introduced in Section 3.4. Therefore, the test statistic for the $i^{\text {th }}$ sample becomes $T_{i}^{2}=\left(\boldsymbol{s}_{i}-\overline{\boldsymbol{s}}\right)^{T} \boldsymbol{\Sigma}^{-1}\left(\boldsymbol{s}_{i}-\overline{\boldsymbol{s}}\right)$, namely, the Hotelling $T^{2}$ statistic. Here, the sample mean $\overline{\boldsymbol{s}}$ and sample covariance matrix $\boldsymbol{\Sigma}$ are estimated from the data. The upper control limit for the Hotelling $T^{2}$ statistic is $U C L=\frac{p(M+1)(M-1)}{M(M-p)} F_{\alpha, p, M-p}$, where $F_{\alpha, p, M-p}$ is the upper $100 \alpha \%$ point of $F$ distribution with $p$ and $M-p$ degrees of freedom.

However, it may be noted that the transition probability between two sub-regions can be zero (see Fig. 7). As such, the sample covariance matrix $\boldsymbol{\Sigma}$ will be singular and it is impractical to calculate the inversion. To address this problem, the feature matrix $\boldsymbol{S}_{M \times p}$ is projected into the eigen-space and a set of linearly uncorrelated principal components are obtained as surrogates. Specifically, the feature matrix $\boldsymbol{S}_{M \times p}$ is centered by subtracting column means, i.e., $\boldsymbol{S}^{*}=\left[\boldsymbol{s}_{1}-\overline{\boldsymbol{s}}, \boldsymbol{s}_{2}-\overline{\boldsymbol{s}}, \ldots, \boldsymbol{s}_{M}-\overline{\boldsymbol{s}}\right]$. Then, the singular value decomposition of $\boldsymbol{S}^{*}$ is computed as $\boldsymbol{S}^{*}=\boldsymbol{U} \boldsymbol{\Psi} \boldsymbol{V}^{T}$, where $\boldsymbol{U}$ and $\boldsymbol{V}$ are $M \times M$ and $p \times p$ orthogonal matrices, $\boldsymbol{\Psi}$ is a $M \times p$ diagonal matrix with diagonal entries $\lambda_{1} \geq \lambda_{2} \geq \cdots \geq \lambda_{p} \geq 0$ (i.e., singular values of $\boldsymbol{S}^{*}$ ). As a result, principal components are given as $\boldsymbol{Z}=\boldsymbol{S}^{*} \boldsymbol{V}=\boldsymbol{U} \boldsymbol{\Psi} \boldsymbol{V}^{\boldsymbol{T}} \boldsymbol{V}=\boldsymbol{U} \boldsymbol{\Psi}=\left[Z_{i k}\right]_{M \times p}$, where $i=1,2, \ldots, M$ is the sample index, $k=1,2, \ldots, p$ is the feature index. Also, the feature matrix can be reconstructed by the principal components as $\boldsymbol{S}^{*}=\boldsymbol{Z} \boldsymbol{V}^{-1}$, because $\boldsymbol{V}$ is an orthogonal matrix and $\boldsymbol{V}^{-1}=\boldsymbol{V}^{T}$. Therefore, the sample covariance matrix $\boldsymbol{\Sigma}$ can be rewritten as $\boldsymbol{\Sigma}=\frac{\boldsymbol{s}^{*} \boldsymbol{S}^{*}}{M-1}=\frac{V \boldsymbol{Z}^{T} \boldsymbol{Z} \boldsymbol{V}^{T}}{M-1}=\boldsymbol{V} \boldsymbol{\Sigma}_{\boldsymbol{Z}} \boldsymbol{V}^{T}$, where $\boldsymbol{\Sigma}_{\boldsymbol{Z}}$ is the covariance matrix with diagonal entries $\lambda_{1}^{2} \geq \lambda_{2}^{2} \geq \lambda_{3}^{2} \geq \cdots \geq \lambda_{p}^{2}$. The Hotelling $T^{2}$ statistic then becomes $T_{i}^{2}=$ $\boldsymbol{Z}_{i} \boldsymbol{V}^{T}\left(\boldsymbol{V} \boldsymbol{\Sigma}_{Z} \boldsymbol{V}^{T}\right)^{-1} \boldsymbol{V} \boldsymbol{Z}_{i}^{T}=\boldsymbol{Z}_{i} \boldsymbol{\Sigma}_{Z}^{-1} \boldsymbol{Z}_{i}^{T}=\frac{\Sigma_{k=1}^{p} Z_{i k}^{p}}{\lambda_{k}^{2}}$, where $\boldsymbol{Z}_{i}$ is the projection of the $i^{\text {th }}$ sample on the principal component directions. To reduce the dimensionality, the first $q$ eigen-values and eigen-vectors $(q<p)$ that explain the majority of variations in the feature matrix are considered. Therefore, the Hotelling $T^{2}$ statistic in the reduced dimension $q$ can be written as $\widetilde{T}^{2}(i)=\sum_{k=1}^{q} \frac{Z_{i k}^{2}}{\lambda_{k}^{2}}$.

\section{Case Studies}

In this section, the proposed methodology are evaluated and validated with two case studies. In the first case study, time series are generated from a simulated Lorenz system, i.e., $\dot{x_{1}}=\sigma\left(x_{2}-x_{1}\right), \dot{x_{2}}=$ $x_{1}\left(\rho-x_{3}\right)-x_{2}, \dot{x_{3}}=x_{1} x_{2}-\beta x_{3}$, where $\left(x_{1}, x_{2}, x_{3}\right)$ is the Lorenz state vector and $\sigma, \rho, \beta$ are the model parameters. Dynamic transitions in the Lorenz system are simulated by varying the parameters. The second case study focuses on the change point detection in the real-world UPM process. Vibration 
signals are collected from the UPM process containing transitions from stable to unstable cutting induced by the onset of chatter, which gives rise to the deterioration of surface finish.

\subsection{Simulation study on the Lorenz system}
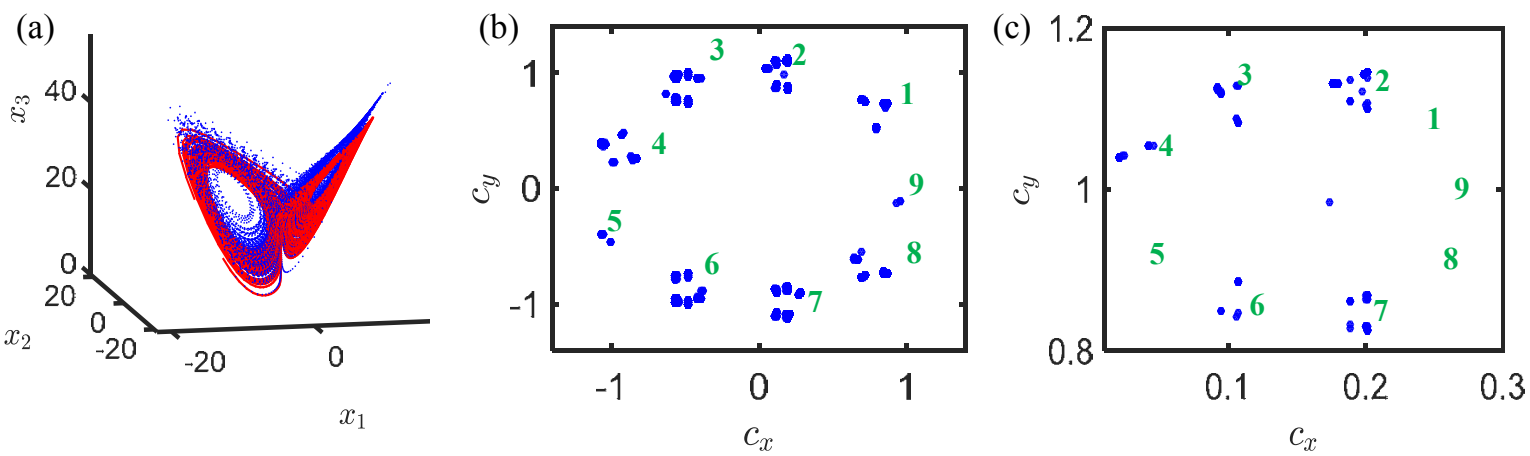

Fig. 8. (a) The state space of Lorenz system with a drift in parameters (from blue: $\sigma=10, \rho=28, \beta=8 / 3$ to red: $\sigma=10, \rho=27, \beta=8 / 3$ ); Fractal representation of state transitions among clusters in (b) in-control system and (c) out-of-control system.

In this simulation study, transitions in the Lorenz system dynamics are simulated by the drift of model parameters. The in-control state space of Lorenz system is simulated by $\sigma=10, \rho=28$, and $\beta=8 / 3$, and the out-of-control state space is generated according to $\sigma=10, \rho=27$, and $\beta=8 / 3$. As shown in Fig. $8 a$, the first 10,000 states (blue dots) represent in-control system dynamics and the next 5,000 states (red dots) are corresponding to out-of-control dynamics. As there is only a slight change in parameter $\rho$, i.e., from 28 to 27 , the blue trajectories and red trajectories in Fig. 8 a are mostly overlapped. It is difficult to discern the variations using traditional time or frequency domain approaches. The DP-driven selforganizing approach partitions the global state space into 9 clusters for heterogeneous recurrence analysis. Fig. $8 \mathrm{~b}$ and $8 \mathrm{c}$ demonstrate patterns of the fractal addresses for in-control states and out-ofcontrol states. Notably, there is a significant difference between the in-control and out-of-control Lorenz system dynamics in the 2D fractal geometry. Here, a sliding window of size $w=1000$ and a step length 50 is constructed to compute the traditional RQA measures and the $T^{2}$ statistics in the multivariate control chart. It may be noted that a threshold $\varepsilon$ is selected to preserve a recurrence rate of $5 \%$ for calculating the recurrence quantifiers.

Further, control charts are constructed for change point detection. First, conventional univariate $\bar{X}$ charts are applied based on traditional RQA measures, i.e., LAM in Fig. 9a and DET in Fig. 9b. As shown in Fig. 9a, both in-control states (e.g., between 6000 to 8000) and out-of-control states (e.g., between 9000 to 11,000 ) get out of the control limits (red dashed lines). This shows that the $\bar{X}$ chart on LAM is limited in the ability to detect the transition in the Lorenz system and it generates large numbers of false alarms. Moreover, no out-of-control is detected from the $\bar{X}$ chart on LAM (see Fig. 9b). This means the univariate chart with traditional RQA measures lacks the detection power to identify slight changes in the dynamic system. In contrast, the multivariate $T^{2}$ chart based on heterogeneous recurrence detects out-ofcontrol states in time and show an early evidence of out-of-control. It may be noted that the blue curve 
gets out of the upper control limit soon after the parameter shift occurs in the Lorenz system. By comparing Fig. 9a and 9b with Fig. 9c, it demonstrates the effectiveness of the proposed recurrence $T^{2}$ chart for detecting dynamical transitions of nonlinear processes, which is superior to the traditional RQA measures DET and LAM.
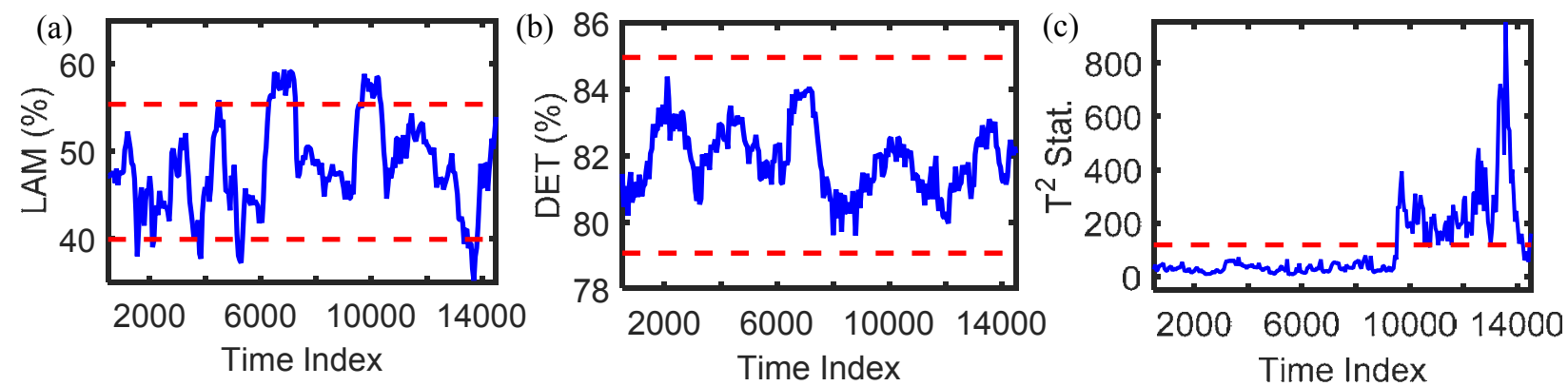

Fig. 9. Univariate $\bar{X}$ chart with traditional RQA measures: (a) LAM and (b) DET; (c) Multivariate $T^{2}$ chart based on heterogeneous recurrence features.

\subsection{UPM processes}

In this case study, the proposed algorithm is evaluated and validated using real-world data collected from UPM processes. It may be noted that the surface morphology of UPM may contain sudden and abrupt variations even manufactured from a well-controlled process. Early detection of process variations can prevent rework and significantly reduce costs [1, 2]. Thus, there is an urgent need to develop efficient schemes for the detection of changes in dynamic regimes (e.g., from stable to unstable cutting) of UPM processes. The experimental setup is shown in Fig. 1. A polycrystalline diamond cutting tool with nose radius of $60 \mu \mathrm{m}$ is used for facing flat surfaces of aluminum workpieces. Three Kistler 8728A500 vibration sensors are placed along three orthogonal directions of the tool holder to measure vibration signals and a Kistler 3-axis dynamometer is mounted on the underside of the tool holder to measure force signals. In this present study, only the vibration signal in the feed direction is considered. As it is suggested in [1], vibrations in the feed direction contribute most to surface roughness variations in UPM processes.

The collected vibration signal in feed direction is shown in Fig. 10a. In the first period, i.e., from the beginning to time index 10000 , the system is stable. After time index 10000 , the system has evolved into an unstable cutting regime (the change point is represented by the vertical red line) due to the onset of chatter. Also known as self-excited vibration, chatter is resulted from the delayed vibration of the cutting tools on the workpiece surface. Chatter may lead to fierce tool vibration and large cutting forces, resulting in rough surfaces and tool damage. Fig. 10b shows a microscopic image of smooth surface obtained from stable cutting. It is with a small surface roughness $(R a=35 \mathrm{~nm})$. After transiting into unstable cutting regime, the surface finish deteriorates and rough surfaces are produced. Fig. 10c shows one example of rough surface $(\mathrm{Ra}=82 \mathrm{~nm})$ with a scratch. With the advancement of machine design, UPM system generally shows high stiffness and damping. As such, the vibration of machine tools is largely suppressed even with the presence of chatter-induced unstable cutting. Consequentially, it is rather difficult to discern 
the stable and unstable periods directly from the time-domain vibration signal in Fig. 10a. Also, vibration signals from these two periods share the same frequency spectrum as illustrated in Fig. 2 . Therefore, it is important to investigate nonlinear dynamics in the process by utilizing recurrence analysis.

(a)

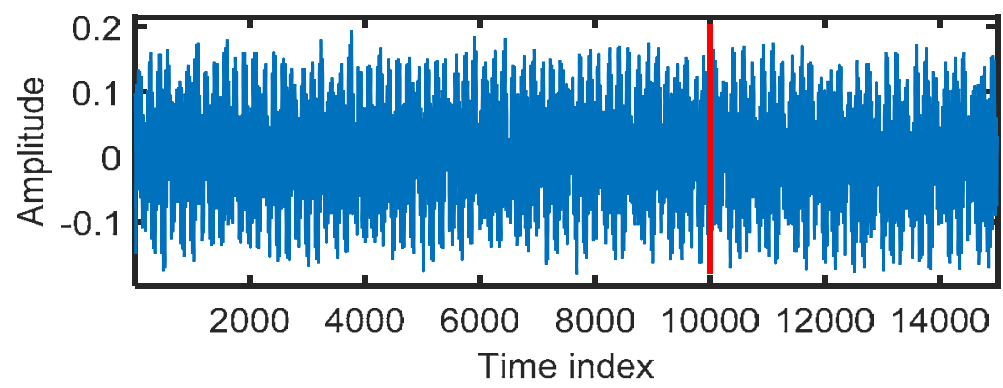

(b)

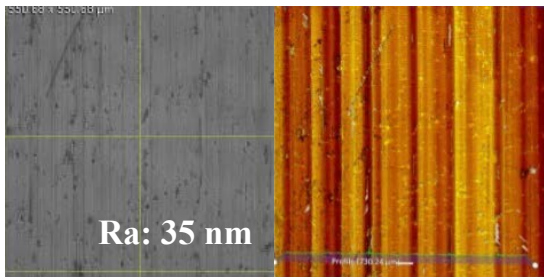

(c)

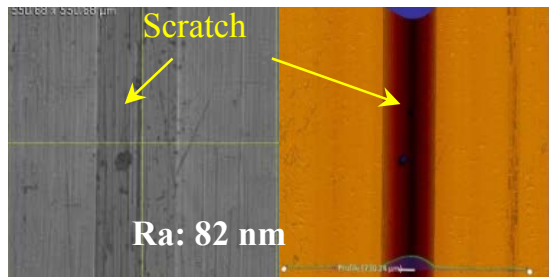

Fig.10. (a) Vibration signal in feed direction with transition from stable to unstable cutting period at time index of 10000 (marked by a red line); (b) Smooth surface (surface roughness $=35 \mathrm{~nm}$ ) obtained from stable cutting; (c) Rough surface with scratches (surface roughness $=82 \mathrm{~nm}$ ) obtained from unstable cutting.

Here, a 3D state space is reconstructed from the $1 \mathrm{D}$ vibration signal with $\tau=5$ and $d=3$, which is then partitioned into eight sub-regions using the developed DP-driven self-organizing approach. The IFS addresses of individual clusters are shown in Fig. 11. It may be note that transitions of individual states in stable and unstable cutting conditions are not clearly distinguished. This explains why the homogeneous recurrence analysis fails to effectively detect the changes. However, by zooming into address 2 in Fig. $11 \mathrm{a}$ and $11 \mathrm{c}$, corresponding 2-state transition patterns of fractal representation are obtained as shown in Fig. $11 \mathrm{~b}$ and $11 \mathrm{~d}$, respectively. A clear difference may be noted from the 2-state transition patterns between stable cutting (Fig. 11b) and unstable cutting (Fig. 11d). For example, the density of points in circles from unstable cutting is smaller than that from stable cutting. This shows the probability of transitions between some sub-regions are significantly reduced in the unstable cutting. 

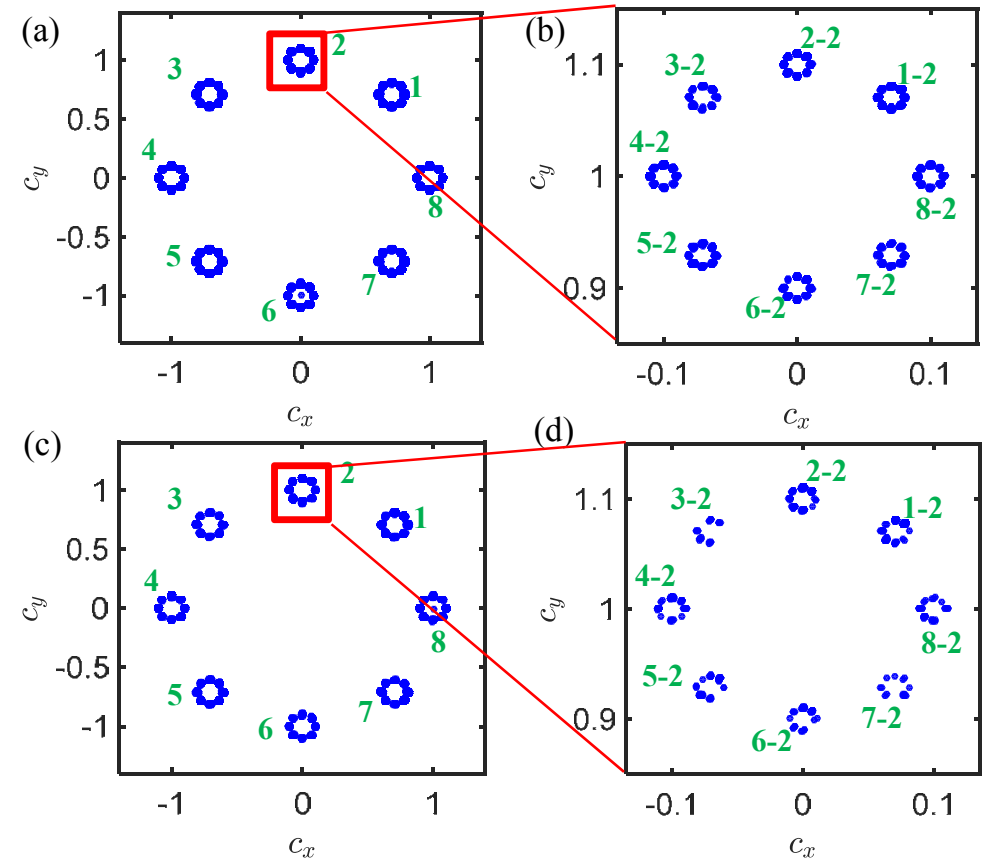

Fig. 11. Fractal representations for (a) individual states and (b) 2-state transition (zooming into address 2 in (a)) under stable cutting conditions; Fractal representation for (c) individual states and (d) 2-state transition (zooming into address 2 in (c)) under unstable cutting conditions.

Further, the performance of proposed heterogeneous recurrence approach is compared with conventional charts. As shown in Fig. $12 \mathrm{a}$ and $12 \mathrm{~b}$, no out-of-control is detected from the $\bar{X}$ charts using traditional RQA measures LAM and DET. This is because the transient changes induced by unstable cutting are more concerned with heterogeneous recurrence variations, whereas assumption of homogeneous recurrence in traditional RQA hampers the detection of nonlinear dynamic transitions. Fig. 12c demonstrates the proposed multivariate $T^{2}$ chart with heterogeneous recurrence. It may be noted that $T^{2}$ statistics get out of the upper control limit as soon as the onset of unstable cutting (at time 10000, shown by a vertical line).
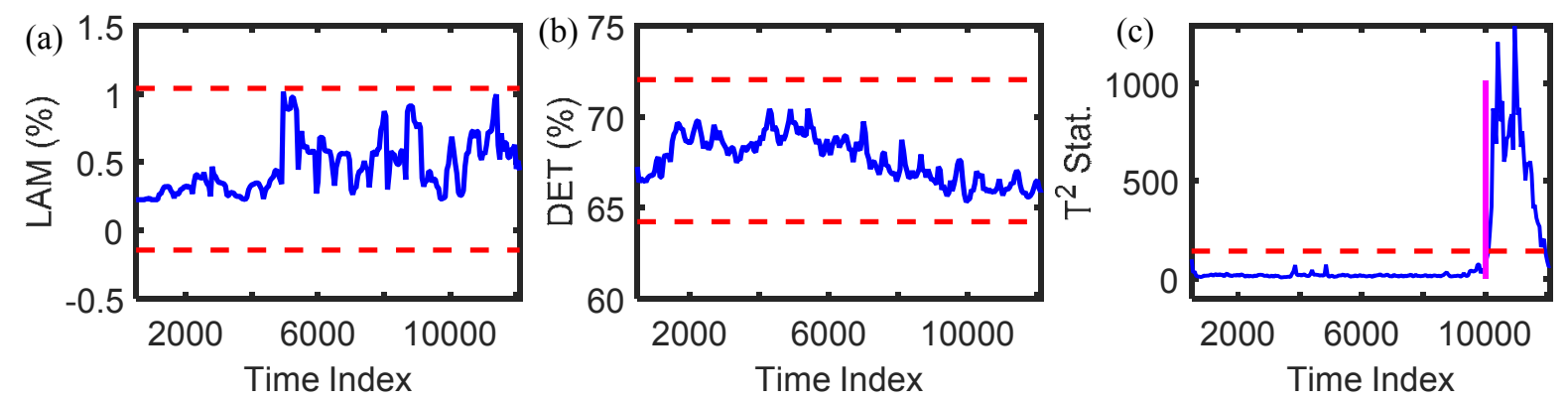

Fig. 12. Univariate $\bar{X}$ chart with traditional RQA measures: (a) LAM and (b) DET; (c) Multivariate $T^{2}$ chart on heterogeneous recurrence features. The vertical line represents the change from stable to unstable cutting. 


\section{Conclusions}

Rapid advancement of sensing technology prompts the sensor-based monitoring and in-situ assessment of surface integrity in the UPM process. As opposed to conventional machining, UPM achieves surface roughness in the range of nanometers and is highly sensitive to the variations of environmental conditions. In-situ sensor signals collected from the UPM process, therefore, manifest nonlinear and nonstationary dynamics. Traditional statistical control approaches are rooted in linear and Gaussian assumptions, and are limited in their ability to detect transient changes resulted from the underlying process dynamics. Traditional recurrence approaches focus on the quantification of homogeneous recurrence behaviors in the UPM process, which register better performance than traditional time and frequency analysis. However, transient dynamics in the UPM processes are more concerned with heterogeneous recurrence variations in the sensor signals. Few, if any, previous approaches have investigated heterogeneous recurrence properties based on the sensor signals for insitu UPM process monitoring and quality control. This paper presents a new heterogeneous recurrence monitoring approach to detect transient changes in UPM. First, a high-dimensional state space is reconstructed from in-situ sensing signals. A Dirichlet process driven clustering approach is then developed to automatically partition the state space into local recurrence regions. Furthermore, a fractal representation is developed to characterize state transitions among recurrence regions and extract novel measures to quantify local heterogeneous recurrence patterns. Finally, a multivariate control chart is designed for on-line monitoring and anomaly detection of UPM processes.

The proposed methodology is first evaluated on a simulated Lorenz system. In the simulation study, only a small drift (i.e., from $\rho=28$ to $\rho=27$ ) occurs in the Lorenz system. Conventional $\bar{X}$ charts on traditional homogeneous recurrence measures either fail to detect the change point or trigger large numbers of false alarms. In contrast, the proposed approach is effective in detecting dynamical transitions of nonlinear processes. The heterogeneous recurrence $T^{2}$ chart yields an early detection of an out-ofcontrol alarm. Second, the proposed approach is evaluated using vibration sensor signals collected from the UPM process. The fractal representation of 2-state transitions demonstrates distinct patterns between stable cutting (in-control) and unstable cutting (out-of-control) in the vibration signals. Again, $T^{2}$ statistics extracted from heterogeneous recurrence show better performances than existing methods. Conventional $\bar{X}$ charts with homogeneous recurrence measures fail to detect the change point, whereas the proposed $T^{2}$ chart signals with the average run length of 1 when the process shift occurs. It is noteworthy that the present investigation provides a novel data-driven DP clustering approach to characterize heterogeneous recurrence variations and link with the quality of surface finishes in UPM processes. This new DP recurrence approach circumvents the need to empirically define local recurrence regions and is shown to have strong potentials for manufacturing process monitoring and control that will increase the surface integrity and reduce rework rates. This investigation assumes sensors are adequate to capture the

intrinsic characteristics of the underlying process. In other words, sensors have no fault and satisfy the 
Nyquist-shannon sampling theorem. Our future investigation will focus on fusing multi-channel signals in the UPM process such as vibration, cutting force and acoustic emission. Each channel provides a different perspective of the process dynamics and the fusion may improve the monitoring performance.

\section{Acknowledgement}

This work is supported in part by the National Science Foundation (CMMI-1646660, CMMI-1617148, CMMI-1619648, and IOS-1146882). The authors also thank Harold and Inge Marcus Career Professorship (HY) for additional financial support.

\section{References}

[1] P. Rao, S. T. S. Bukkapatnam, O. Beyca, Z. Kong and R. Komanduri, "Real-time identification of incipient surface morphology variations in ultraprecision machining process," ASME. J. Manuf. Sci. Eng., vol. 136, No.2, pp. 021008-11, 2014.

[2] O. Beyca, P. Rao, Z. Kong, S. Bukkapatnam and R. Komanduri, "Heterogeneous sensor data fusion approach for real-time monitoring in ultraprecision machining (UPM) process using non-parametric Bayesian clustering and evidence theory," Automation Science and Engineering, IEEE Transactions on, vol. 13, No.2, pp. 1033-1044, 2016.

[3] S. Takasu, M. Masuda and T. Nishiguchi, "Influence of study vibration with small amplitude upon surface roughness in diamond machining," CIRP Ann., vol. 34, pp. 463-467, 1985.

[4] C. Cheung and W. Lee, "Modeling and simulation of surface topography in ultraprecision diamond turning," Proc Instn Mech Engrs, vol. 214, pp. 463-480, 2000.

[5] D. Dornfeld, Y. Lee and A. Chang, "Monitoring of ultraprecision machining process," Int. J. Adv. Manuf. Technol., vol. 21, pp. 571-578, 2003.

[6] D. Dornfeld, "Process monitoring and control for precision manufacturing," Prod. Eng., vol. 6, pp. 2934, 1999.

[7] S. Bukkapatnam, A. Lakhtakia and S. Kumara, "Analysis of sensor signals shows turning on a lathe exhibits low-dimensional chaos," Physical Review E, vol. 52, No.3, pp. 2375-2387, 1995.

[8] S. Bukkapatnam, S. Kamarthi, Q. Huang, A. Zeid and R. Komanduri, "Nanomanufacturing systems: opportunities for industrial engineers," IIE Transactions, vol. 44, No.7, pp. 492-495, 2012.

[9] Z. Wang, S. Bukkapatnam, S. Kumara, Z. Kong and Z. Katz, "Change detection in precision manufacturing processes under transient conditions," CIRP Annals - Manufacturing Technology, vol. 63, pp. 449-452, 2014.

[10] P. Rao, O. Beyca, Z. Kong, S. Bukkapatnam, K. Case and R. Komanduri, "A graph-theoretic approach for quantification of surface morphology variation and its application to chemical mechanical planarization process," IIE Transactions, vol. 47, No.10, pp. 1088-1111, 2015.

[11] S. Bukkapatnam and C. Cheng, "Forecasting the evolution of nonlinear and nonstationary systems using recurrence-based local Gaussian process models," Physical Review E, vol. 82, pp. 056206, 2010.

[12] N. Marwan, M. Carmen Romano, M. Thiel and J. Kurths, "Recurrence plots for the analysis of complex systems," Physics Reports, vol. 438, No.5-6, pp. 237-329, 1, 2007. 
[13] H. Yang, "Multiscale recurrence quantification analysis of spatial cardiac vectorcardiogram signals," Biomedical Engineering, IEEE Transactions on, vol. 58, No.2, pp. 339-347, 2011.

[14] Y. Chen and H. Yang, "Multiscale recurrence analysis of long-term nonlinear and nonstationary time series," Chaos, Solitons and Fractals, vol. 45, pp. 978-987, 2012.

[15] Y. Chen and $\mathrm{H}$. Yang, "Heterogeneous recurrence monitoring and control of nonlinear stochastic processes," Chaos, vol. 24, No.1, pp. 013138, 2014.

[16] Y. Chen and $\mathrm{H}$. Yang, "Heterogeneous recurrence representation and quantification of dynamic transitions in continuous nonlinear processes," Eur. Phys. J. B, vol. 89, pp. 155, 2016.

[17] C. Cheng, C. Kan and H. Yang, "Heterogeneous recurrence analysis of heartbeat dynamics for the identification of sleep apnea events," Computers in Biology and Medicine, vol. 75, pp. 10-18, 2016.

[18] H. Yang, S. Bukkapatnam and R. Komanduri, "Spatiotemporal representation of cardiac vectorcardiogram (VCG) signals," Biomedical Engineering Online, vol. 11, No.12, 2012.

[19] C. Cheng, A. Sa-Ngasoongsong, O. Beyca, et al, "Time series forecasting for nonlinear and nonstationary processes: a review and comparative study," IIE Transactions, vol. 47, No.10, pp. 10531071, 2015.

[20] P. Rao, S. Bukkapatnam, O. Beyca, A. Fields, Z. Kong and R. Komanduri, "Process-machine interaction (PMI) modeling and monitoring of chemical mechanical planarization (CMP) process using wireless vibration sensors," Semiconductor Manufacturing, IEEE Transactions on, vol. 27, No.1, pp. 1-15, 2014.

[21] G. Singer and I. Ben-Gal, "The funnel experiment: The Markov-based SPC approach," Quality and Reliability Engineering International, vol. 23, pp. 899-913, 2007.

[22] N. Ruschin-Riminia, I. Ben-Gal and O. Maimona, "Fractal geometry statistical process control for nonlinear pattern-based processes," IIE Transactions, vol. 45, No.4, pp. 355-373, 2013.

[23] Y. Chen and $\mathrm{H}$. Yang, "Heterogeneous recurrence $\mathrm{T}^{2}$ charts for monitoring and control of nonlinear dynamic processes," in Proc. 11th Ann. IEEE Int. Conf. Autom. Sci. Eng. (CASE 2015), Gothenburg, Sweden, Aug. 24-28, 2015.

[24] H. Yang, S. Bukkapatnam and L. Barajas, "Local recurrence based performance prediction and prognostics in the nonlinear and nonstationary systems," Pattern Recognit, vol. 44, No.8, pp. 18341840, 8, 2011.

[25] M. Valipour, "Optimization of neural networks for precipitation analysis in a humid region to detect drought and wet year alarms," Meteorological Applications, vol. 23, No.1, pp. 91-100, 2016.

[26] M. Valipour, M. Sefidkouhi and S. Eslamian, "Surface irrigation simulation models: a review," Int. J. Hydrology Science and Technology, vol. 5, No.1, pp. 51-70, 2015.

[27] D. Blei and M. Jordan, "Variational inference for Dirichlet process mixtures," Bayesian Analysis, vol. 1, pp. 121-144, 2005. 